\title{
The Role of the Intergeniculate Leaflet in Entrainment of Circadian Rhythms to a Skeleton Photoperiod
}

\author{
Kim Edelstein and Shimon Amir \\ Center for Studies in Behavioral Neurobiology, Department of Psychology, Concordia University, Montreal, Quebec, \\ Canada H3G 1 M8
}

\begin{abstract}
Mammalian circadian rhythms are synchronized to environmental light/dark (LD) cycles via daily phase resetting of the circadian clock in the suprachiasmatic nucleus (SCN). Photic information is transmitted to the SCN directly from the retina via the retinohypothalamic tract $(\mathrm{RHT})$ and indirectly from the retinorecipient intergeniculate leaflet (IGL) via the geniculohypothalamic tract (GHT). The RHT is thought to be both necessary and sufficient for photic entrainment to standard laboratory light/ dark cycles. An obligatory role for the IGL-GHT in photic entrainment has not been demonstrated. Here we show that the IGL is necessary for entrainment of circadian rhythms to a skeleton photoperiod (SPP), an ecologically relevant lighting schedule congruous with light sampling behavior in nocturnal rodents. Rats with bilateral electrolytic IGL lesions entrained normally to lighting cycles consisting of $12 \mathrm{hr}$ of light followed
\end{abstract}

by $12 \mathrm{hr}$ of darkness, but exhibited free-running rhythms when housed under an SPP consisting of two $1 \mathrm{hr}$ light pulses given at times corresponding to dusk and dawn. Despite IGL lesions and other damage to the visual system, the SCN displayed normal sensitivity to the entraining light, as assessed by lightinduced Fos immunoreactivity. In addition, all IGL-lesioned, free-running rats showed masking of the body temperature rhythm during the SPP light pulses. These results show that the integrity of the IGL is necessary for entrainment of circadian rhythms to a lighting schedule like that experienced by nocturnal rodents in the natural environment.

Key words: suprachiasmatic nucleus; neuropeptide Y; immediate early gene; geniculohypothalamic tract; retinohypothalamic tract; body temperature
Environmental light is necessary for stable entrainment of mammalian circadian rhythms, providing the critical cue for daily resetting of the circadian clock in the suprachiasmatic nucleus of the hypothalamus (SCN) (Pittendrigh and Daan, 1976; Moore, 1983; Morin, 1994; Roenneberg and Foster, 1997). Light presented near dusk delays the phase of the clock, whereas light presented near dawn advances the phase of the clock (Daan and Pittendrigh, 1976; Roenneberg and Foster, 1997). Photic information is transmitted to the SCN via two principal pathways: a direct retinal projection, the retinohypothalamic tract (RHT), and an indirect projection originating in the retinorecipient intergeniculate leaflet (IGL), the geniculohypothalamic tract (GHT) (Card and Moore, 1991; Moore and Card, 1994; Morin, 1994; Harrington, 1997). The RHT is thought to be both necessary and sufficient for photic entrainment. Disruption of the RHT, but not other retinofugal projections, prevents photic entrainment to standard laboratory light/dark (LD) cycles (Dark and Asdourian, 1975; Pickard et al., 1987; Harrington and Rusak, 1988; Johnson et al., 1988, 1989). In contrast, a role for the IGL-GHT in the circadian response to light has not been well established. Current evidence suggests that the IGL modulates the rate of re-entrainment to shifted LD cycles (Johnson et al., 1989; Harrington, 1997) and the circadian period during constant

\footnotetext{
Received Sept. 8, 1998; revised Oct. 2, 1998; accepted Oct. 12, 1998.

This work was supported by grants from the Medical Research Council of Canada, the Natural Sciences and Engineering Research Council of Canada, and the Fonds pour la Formation de Chercheurs et l'Aide à la Recherche (Québec). We thank Jane Stewart for helpful comments on this manuscript.

Correspondence should be addressed to Shimon Amir, Center for Studies in Behavioral Neurobiology, Department of Psychology, Concordia University, 1455 de Maisonneuve Boulevard West H-1013, Montreal, Quebec, Canada H3G 1M8. Copyright (C) 1998 Society for Neuroscience 0270-6474/98/190372-09\$05.00/0
}

light housing in hamsters (Pickard et al., 1987; Harrington and Rusak, 1988; Harrington, 1997) but not mice (Pickard, 1994) or rats (Edelstein and Amir, 1999). IGL lesions, however, do not prevent entrainment to standard laboratory LD cycles (Pickard et al., 1987; Harrington and Rusak, 1988; Johnson et al., 1989; Moore and Card, 1994; Harrington, 1997; Edelstein and Amir, 1999), suggesting only a minor contribution of the GHT to the photic entrainment process. There is evidence, however, that IGL lesions alter the phase angle of entrainment under a sinuosoidal lighting schedule (Pickard, 1989), consistent with Pickard's idea (Pickard et al., 1987) that the IGL is involved in photic entrainment under natural lighting conditions.

A possible role for the IGL in light-induced resetting of the circadian clock is further supported by recent evidence demonstrating that neuropeptide Y (NPY), a neurotransmitter used by the GHT (Moore and Card, 1994; Harrington, 1997), exhibits a diurnal rhythm in the SCN with peaks occurring at the time of light/dark transitions at dawn and dusk (Shinohara et al., 1993). NPY has been found to depress excitatory neurotransmission in the SCN (van den Pol et al., 1996) and to block glutamateinduced phase shifts in SCN neuronal activity in vitro (Biello et al., 1997) and light-induced phase advances in vivo (Weber and Rea, 1997). These findings raise the possibility that the IGL is involved in synchronization of circadian rhythms to discrete light pulses.

Circadian rhythms of nocturnal animals can be entrained by brief, discrete pulses of light given at dusk and dawn (Pittendrigh and Daan, 1976; Rosenwasser et al., 1983; Stephan, 1983; DeCoursey, 1986). Housing under such a skeleton photoperiod (SPP) provides an ecologically relevant lighting schedule congruous with light-sampling behavior in nocturnal rodents under nat- 
ural and simulated laboratory conditions (DeCoursey, 1986). Moreover, inasmuch as exposure to light can suppress overt expression of some rhythmic variables (Mrosovsky, 1994), SPP housing allows for the study of pacemaker entrainment in the absence of the interfering masking effects of light.

In the present study, we investigated the involvement of the IGL in entrainment of circadian rhythms by assessing the effects of IGL lesions on body temperature rhythms of animals housed under an SPP consisting of two $1 \mathrm{hr}$ light pulses at times corresponding to dusk and dawn. The effect of IGL lesions on the response of the SCN to the entraining light pulses was assessed using expression of the transcription factor Fos as a marker. The integrity of the GHT after IGL lesions was evaluated using immunohistochemical staining of NPY in the SCN.

Preliminary results have been published previously in abstract form (Edelstein and Amir, 1997).

\section{MATERIALS AND METHODS}

Animals. Male Wistar rats (Charles River Canada, St. Constant, Quebec) were used in all experiments. They were housed under $12 \mathrm{hr}$ light/dark cycle (LD12:12) with free access to food and water for at least 2 weeks before surgery.

Surgery. Rats (275-300 gm) were anesthetized with sodium pentobarbital $(65 \mathrm{mg} / \mathrm{kg}$, i.p.) and implanted intraperitoneally with precalibrated telemetry transmitters (Mini Mitter Co., Sunriver, OR). Atropine sulfate $(0.06 \mathrm{mg} / 0.1 \mathrm{ml}$, s.c.) was given $20 \mathrm{~min}$ before anesthesia. Bilateral electrolytic lesions, aimed at the IGL, were made by passing $2 \mathrm{~mA}$ current for $15 \mathrm{sec}$ through stainless steel electrodes, insulated except for the tip (Grass LM4 lesion maker). Electrode placements were at three rostrocaudal positions on each side of the midline: $4.0,4.7$, and $5.4 \mathrm{~mm}$ posterior to bregma, $3.8 \mathrm{~mm}$ lateral to the midsagittal sinus, and $4.7 \mathrm{~mm}$ ventral to dura. These coordinates were determined from IGL lesions produced in test animals. Sham-operated rats were subjected to the same surgical procedures, except that electrodes were lowered to $1 \mathrm{~mm}$ above the lesion targets and no current was passed.

Temperature rhythm recording and data analysis. Rats were housed individually in plastic cages in light-tight, temperature-controlled, ventilated rooms, and body temperature was recorded at $10 \mathrm{~min}$ intervals using a telemetry system. Circadian temperature rhythms were measured under LD12:12 (light intensity at cage level was $~ 300$ lux), SPP, and constant darkness (DD) with Dataquest software (Mini Mitter). The SPP consisted of two $1 \mathrm{hr}$ light pulses $(\sim 300 \mathrm{lux})$ given at zeitgeber times (ZT) corresponding to lights on and lights off of the previous LD cycle (ZT0-1 and ZT11-12, where ZT0 = light onset). This lighting schedule maintained the long night of the previous LD cycle ( $12 \mathrm{hr}$ between light pulses) with a shorter day $(10 \mathrm{hr})$, a regimen that facilitates stable entrainment in nocturnal animals (Pittendrigh and Daan, 1976; Stephan, 1983). The period of the circadian temperature rhythm for each animal was calculated during the first $6 \mathrm{~d}$ of DD housing using cosinor analysis of body temperature data smoothed with a $90 \mathrm{~min}$ moving average, and group means were compared ( $t$ test). Entrainment to LD cycles was assessed by visual inspection of body temperature records, constructed as "actograms" using Dataquest software (Mini Mitter).

Photic stimulation. One group of rats was housed in LD12:12 for 3 weeks and killed after $1 \mathrm{hr}$ of light ( $\sim 300$ lux at ZT1) on day 21-23. A second group of rats was housed in LD12:12 for $7 \mathrm{~d}$ and then released into a SPP. These animals were then killed after either the dawn (ZT0-1) or dusk (ZT11-12) light pulses ( 300 lux) on days 5 or 6 of the SPP. A third group of animals for which rhythms were not recorded was housed under LD12:12 for 3 weeks after surgery, transferred into DD for $24-36 \mathrm{hr}$, and then killed after exposure to a $1 \mathrm{hr}$ light pulse $(\sim 300$ lux $)$ in the middle of the projected subjective day [between circadian time (CT) 3-5, where CT0 = dawn] or night (between CT15 and CT17). Serial brain sections through the SCN were examined under a microscope, and the number of Fos-immunopositive cells was established using a computerized image analysis system consisting of a Sony XC-77 Video Camera, a Scion LG-3 frame grabber, and NIH Image Software. For each animal, the score used was the mean number of Fos-positive cells calculated from the three sections exhibiting the highest number of counts. The effects of time of light exposure (PHASE) and treatment (GROUP) on the number of Fos-immunoreactive cells were evaluated using ANOVA.
Preparation of tissue. Animals were deeply anesthetized with sodium pentobarbital and perfused transcardially with $300 \mathrm{ml}$ of cold physiological saline $(0.9 \% \mathrm{NaCl})$ followed by $300 \mathrm{ml}$ of cold $4 \%$ paraformaldehyde in a $0.1 \mathrm{M}$ phosphate buffer, $\mathrm{pH}$ 7.3. Brains were removed and post-fixed overnight in $4 \%$ paraformaldehyde at $4^{\circ} \mathrm{C}$. Serial coronal brain sections $(50 \mu \mathrm{m})$ through the SCN were cut from each brain on a vibratome, and alternate sections were processed for either NPY or Fos immunohistochemistry. The caudal portions of brains of IGL-lesioned and shamoperated animals were cryoprotected in $30 \%$ sucrose formalin solution, and frozen coronal sections $(30 \mu \mathrm{m})$ through the lateral geniculate nucleus were sliced on a cryostat and stained with thionin.

NPY immunohistochemistry. Tissue sections were washed in cold $50 \mathrm{~mm}$ Tris-buffered saline (TBS), pH 7.6 (Sigma, St. Louis, MO), and incubated in a solution of $30 \% \mathrm{H}_{2} \mathrm{O}_{2}$ (Sigma) in TBS for $30 \mathrm{~min}$ at room temperature. These sections were then incubated in a solution of $0.3 \%$ Triton X-100 (Sigma) in TBS (0.3\% TTBS) with 4\% normal goat serum (NGS) (Vector, Burlingame, CA) on an orbital shaker for $1 \mathrm{hr}$ at room temperature. Tissue sections were then incubated for $48 \mathrm{hr}$ at $4^{\circ} \mathrm{C}$ with a rabbit polyclonal anti-NPY antibody (lot 960108-2; Peninsula, Belmont, CA). The antibody was diluted 1:5000 with a solution of $0.3 \%$ TTBS with $2 \%$ NGS. After incubation in the primary antibody, sections were rinsed in cold TBS and incubated for $1 \mathrm{hr}$ at $4{ }^{\circ} \mathrm{C}$ with a biotinylated anti-rabbit IgG made in goat (Vector), diluted 1:66 with $0.3 \%$ TTBS with $2 \%$ NGS. After incubation with secondary antibody, sections were rinsed in cold TBS and incubated for $2 \mathrm{hr}$ at $4^{\circ} \mathrm{C}$ with an avidin-biotin-peroxidase complex (Vectastain Standard Elite ABC Kit, Vector). After incubation with the $\mathrm{ABC}$ reagents, sections were rinsed with cold TBS, rinsed again with cold $50 \mathrm{~mm}$ Tris buffer, $\mathrm{pH}$ 7.6, and again for $10 \mathrm{~min}$ with $0.05 \%$ $3,3^{\prime}$-diaminobenzidine (DAB) (Sigma) in $50 \mathrm{~mm}$ Tris-HCl. Sections were then incubated on an orbital shaker for $10 \mathrm{~min}$ in $\mathrm{DAB} / \mathrm{Tris}-\mathrm{HCl}$ with $0.01 \% \mathrm{H}_{2} \mathrm{O}_{2}$ and $8 \% \mathrm{NiCl}_{2}$ (Sigma). After this final incubation, sections were rinsed with cold TBS, wet-mounted onto gel-coated slides, dehydrated through a series of alcohols, soaked in xylene, and coverslipped with Permount (Fisher).

Fos immunohistochemistry. Tissue sections were washed in cold $50 \mathrm{~mm}$ TBS and incubated for $48 \mathrm{hr}$ at $4^{\circ} \mathrm{C}$ with a mouse monoclonal antibody raised against the $\mathrm{N}$-terminal sequence of Fos (corresponding to $\mathrm{N}$-terminal residues 4-17 of human Fos protein, lot number 411-081887; NCI/BCB Repository, Quality Biotech, Camden, NJ). This antibody produces one band on Western blots with a molecular weight characteristic of Fos and is therefore believed to recognize Fos protein but not Fos-related antigens (de Togni et al., 1988). Blocking experiments performed by exposing tissue sections to the N-terminal Fos peptide (2 $\mu \mathrm{g} / \mathrm{ml}$ ) in the primary antibody incubation solution prevents Fos-IR staining, as described previously (Edelstein and Amir, 1996). The antibody was diluted 1:8000 with a solution of $0.3 \%$ TTBS with $1 \%$ normal horse serum (NHS) (Vector). After incubation in the primary antibody, sections were rinsed in cold TBS and incubated for $1 \mathrm{hr}$ at $4^{\circ} \mathrm{C}$ with a rat-adsorbed biotinylated anti-mouse IgG made in horse (Vector), diluted 1:33 with $0.3 \%$ TTBS with $1 \%$ NHS. After incubation with secondary antibody, sections were rinsed in cold TBS and incubated for $2 \mathrm{hr}$ at $4^{\circ} \mathrm{C}$ with $\mathrm{ABC}$ reagents (Vector). Sections were then rinsed with cold TBS, rinsed again with cold $50 \mathrm{~mm}$ Tris buffer, $\mathrm{pH}$ 7.6, and again for 10 min with $0.05 \%$ DAB in $50 \mathrm{~mm}$ Tris- $\mathrm{HCl}$. Sections were then incubated on an orbital shaker for $10 \mathrm{~min}$ in $\mathrm{DAB} / \mathrm{Tris}-\mathrm{HCl}$ with $0.01 \% \mathrm{H}_{2} \mathrm{O}_{2}$ and $8 \% \mathrm{NiCl}_{2}$. After this final incubation, sections were rinsed with cold TBS, wet-mounted onto gel-coated slides, dehydrated through a series of alcohols, soaked in xylene, and coverslipped with Permount (Fisher).

Histology. Completeness of IGL lesions was verified under a microscope using two criteria: absence of NPY fiber staining in the SCN and inspection of thionin-stained sections through the lateral geniculate nucleus (LGN).

\section{RESULTS}

All lesioned animals sustained large lesions centered on the IGL, with extensive damage to the lateral geniculate complex, as well as part of the hippocampus and the optic tract. Schematic drawings of the largest and smallest lesion are shown in Figure 1. Functional effectiveness of the lesion was confirmed by the absence of NPY immunoreactivity (NPY-IR) in the SCN (Fig. 2). 


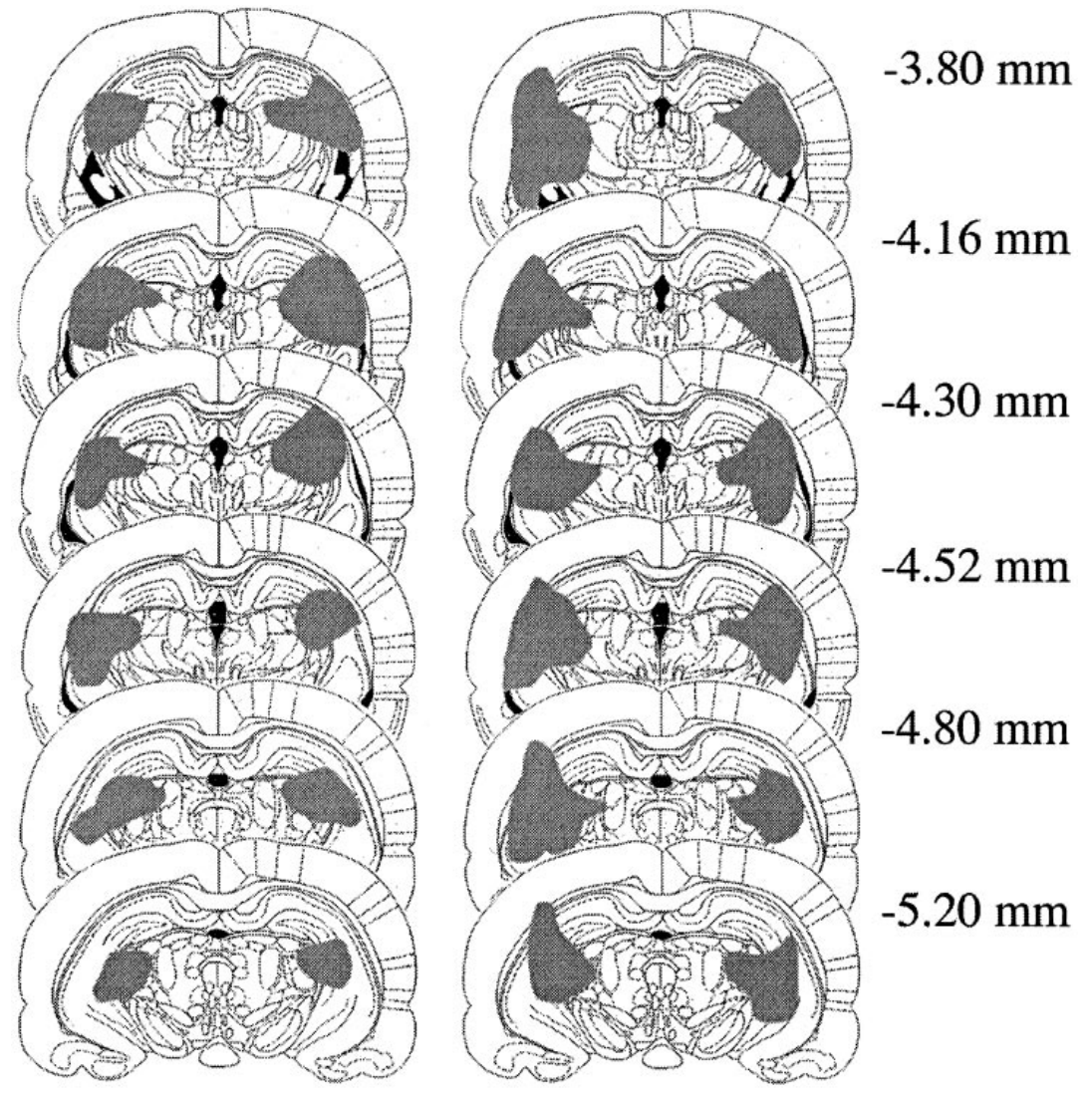

Figure 1. Largest (right) and smallest (left) electrolytic lesions centered on the intergeniculate leaflet in six coronal sections at the levels indicated in millimeters posterior to bregma (from Paxinos and Watson, 1998).

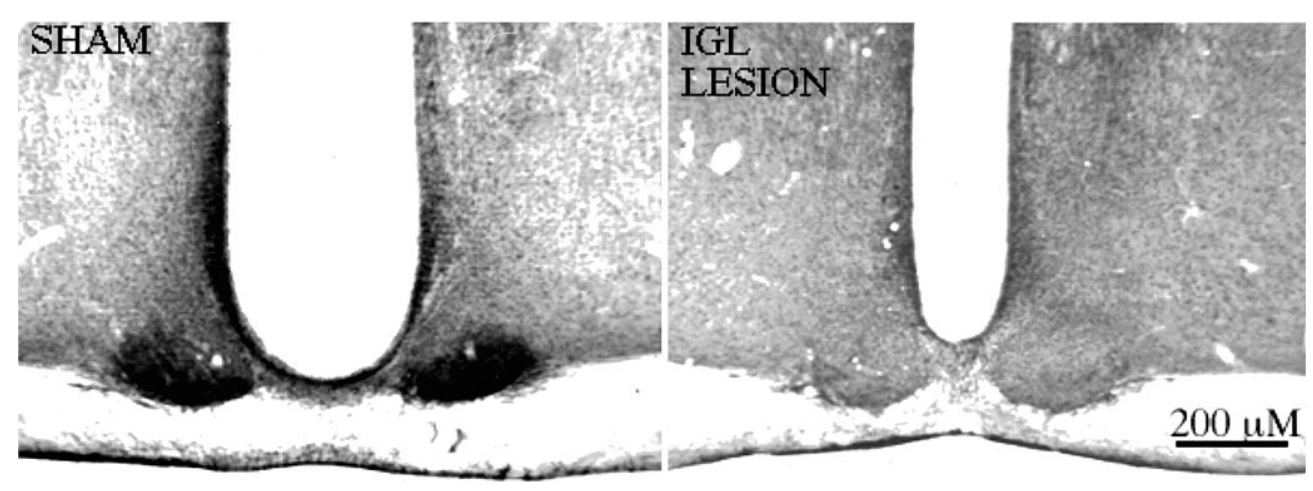

Figure 2. Neuropeptide Y immunoreactivity in coronal sections through the SCN of sham-operated (left) and IGL-lesioned (right) rats. Images were digitized using a computerized image analysis system using a Sony XC-77 Video Camera, a Scion LG-3 frame grabber, and NIH Image Software.

\section{Experiment 1: Circadian temperature rhythms during LD12:12, SPP, and DD}

In this experiment IGL-lesioned $(n=7)$ and sham-operated $(n=$ 3) animals were housed under LD12:12 for $14 \mathrm{~d}$, followed by 3 weeks of SPP, and were then released into DD for 2 weeks. Two lesioned animals were eliminated from the analysis because they exhibited $24 \mathrm{hr}$ free-running periods, making it impossible to confirm entrainment. The remaining animals entrained to LD12:12 and exhibited free-running rhythms under DD housing conditions. All animals had free-running periods slightly greater than $24 \mathrm{hr}$ in DD (Table 1), and no significant difference was found between IGL-lesioned and sham-operated animals $\left(t_{(8)}=\right.$ 0.363 , NS). Under the SPP, rhythms of sham-operated rats remained entrained. In contrast, four of the five rats with IGL lesions exhibited free-running rhythms. Sample temperature records of an IGL-lesioned and a sham-operated rat during LD12:12, SPP, and DD housing are shown in Figure 3.

\section{Experiment 2: Circadian temperature rhythms during prolonged SPP housing}

This experiment was performed to determine whether the failure of IGL-lesioned rats to entrain to the SPP (Experiment 1) merely reflected sluggish adjustment of the clock to this novel lighting schedule that could be overcome with time. It was suspected that lesioned animals might be able to establish stable entrainment if allowed to free run for a period of time sufficient to permit the light pulses of the SPP to fall at times when light could effectively entrain the rhythm to a $24 \mathrm{hr}$ schedule. This idea was tested by measuring temperature rhythms of IGL-lesioned $(n=7)$ and sham-operated $(n=4)$ rats housed under LD12:12 and then 
Table 1. Period of the body temperature rhythm of IGL-lesioned and sham-operated rats in DD

\begin{tabular}{lll} 
& $\begin{array}{l}\text { Mean } \\
\text { (hours } \pm \text { SEM) }\end{array}$ & Range (hr: min-max) \\
\hline Sham $(n=3)$ & $24.28 \pm 0.07$ & $24.17-24.42$ \\
IGL-lesion $(n=7)^{a}$ & $24.33 \pm 0.09$ & $24.08-24.75$
\end{tabular}

${ }^{a}$ Includes data from two IGL-lesioned animals that exhibited a $24 \mathrm{hr}$ free-running period.

released into an SPP for $48 \mathrm{~d}$. One sham-operated animal was eliminated from the analysis because it exhibited a $24 \mathrm{hr}$ freerunning period. The remaining sham-operated animals maintained steady phase relationships with the SPP throughout this period. Of these, one was entrained throughout the 7 week period; one animal phase-jumped after $30 \mathrm{~d}$ in the SPP so that the active phase became the shorter period between the two light pulses, and the third animal entrained for $21 \mathrm{~d}$, after which it exhibited rapid delay transients before re-entraining to the SPP for the final $15 \mathrm{~d}$ of SPP housing. In contrast, four of the seven IGL-lesioned rats did not entrain, exhibiting free-running rhythms throughout the SPP housing period despite several $360^{\circ}$ revolutions through the SPP. The remaining three IGL-lesioned rats free ran for $27-31 \mathrm{~d}$, re-entraining only after a $360^{\circ}$ revolution in the rhythm. Although IGL-lesioned animals failed to show normal entrainment to the SPP, adjustments in the period of the rhythm as a function of phase of the LD cycle (relative coordination) was observed. Furthermore, masking, or acute suppression, of body temperature during times of light exposure was evident in all animals that failed to entrain to the SPP. This suggests that IGL-lesioned animals continue to respond to acute effects of light on physiological responses such as body temperature despite considerable damage to the lateral geniculate nucleus. The masking effect of light and the relative coordination observed during the SPP in IGL-lesioned animals can be seen in
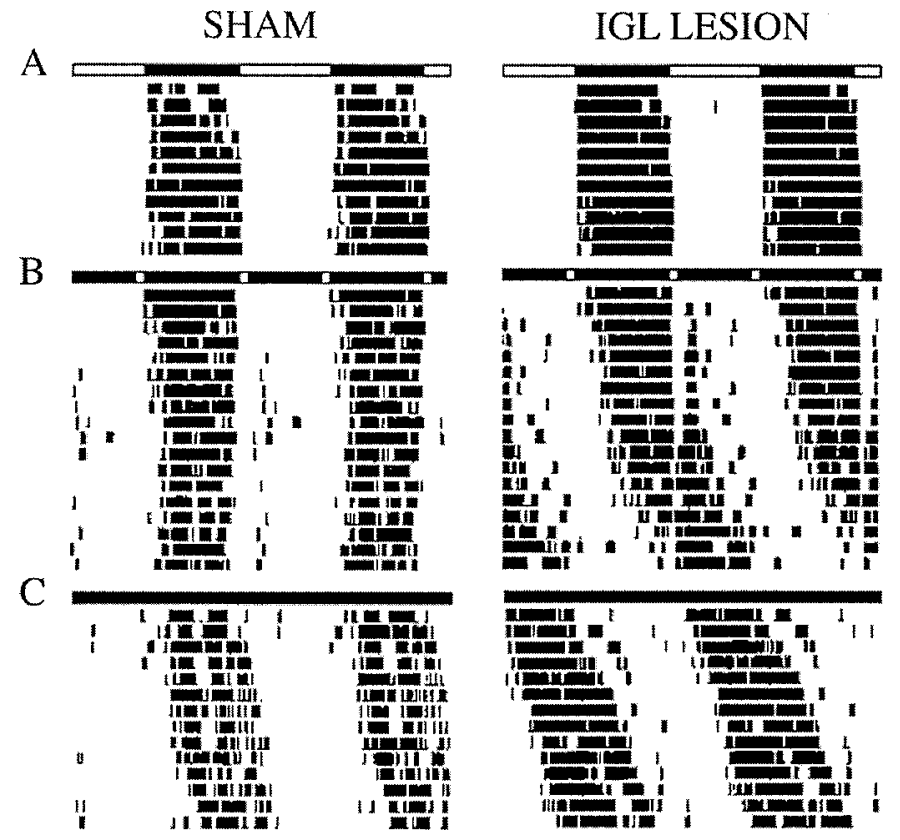

Figure 3. Double-plotted actograms of body temperature records of a sham-operated and an IGL-lesioned rat under LD12:12 $(A), \operatorname{SPP}(B)$, and DD $(C)$. Light bars = lights on; dark bars = lights off.
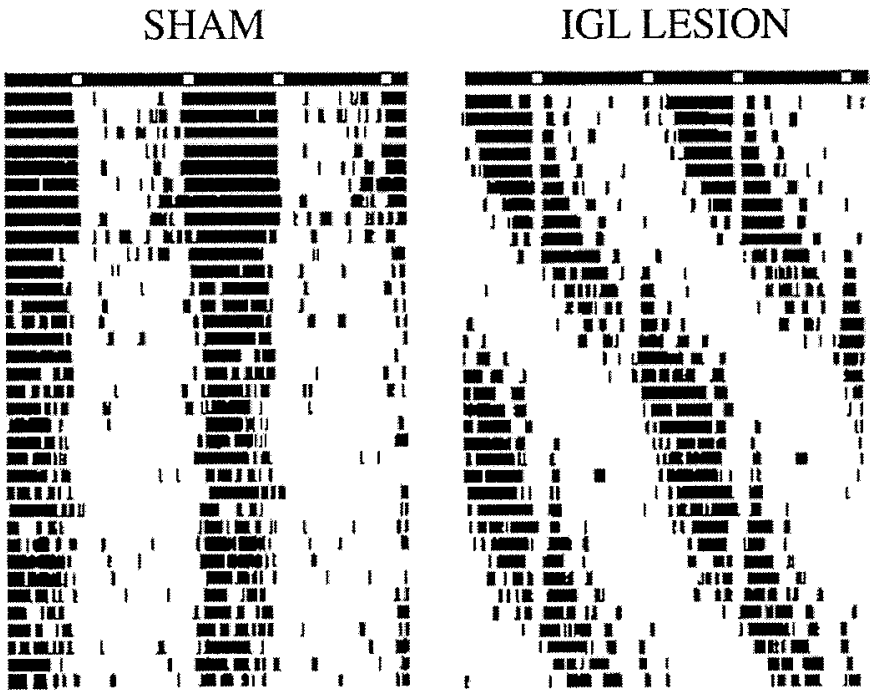

Figure 4. Double-plotted actograms of body temperature records of a sham-operated and an IGL-lesioned rat during prolonged SPP housing. Light bars $=$ lights on; dark bars $=$ lights off.

the temperature records of a representative IGL-lesioned animal shown in Figure 4.

\section{Entrainment or masking under LD12:12?}

Because IGL-lesioned rats exhibited suppression of body temperature during exposure to the light pulse under the SPP, we considered the possibility that the synchronized temperature rhythm observed under LD12:12 might have been caused by the masking effect of light on temperature rather than the entraining effect of light on the clock. To test whether animals were actually entrained to LD12:12, free-running animals housed in DD (from Experiment 2) were placed in LD12:12 for 2 weeks and then released again into DD for $10 \mathrm{~d}$. The time of onset of the free-running rhythm was examined relative to the time of lights off of the previous LD cycle and compared with the predicted onset extrapolated from the initial free run. No differences between IGL-lesioned and sham-operated animals were observed in this test; in all animals, the onset of free running occurred at the time that could be predicted from the LD cycle and not from the initial free run, demonstrating that IGL-lesioned rats entrain to full photoperiods (Fig. 5).

\section{Light-induced Fos immunoreactivity in the SCN}

To test whether the failure of IGL-lesioned rats to entrain to the SPP was caused by disruption of transmission of the entraining light pulses to the SCN, we assessed the profile of light-induced Fos protein expression in the SCN. Fos expression in the SCN is induced by light stimuli that phase shift circadian rhythms, and the magnitude of expression correlates with the effectiveness of light as a resetting stimulus (Rea, 1989; Aronin et al., 1990; Kornhauser et al., 1990; Rusak et al., 1990). To study the effect of light exposure at dawn, animals (from Experiment 1) were housed under LD12:12 and killed at ZT1, $1 \mathrm{hr}$ after light onset. As can be seen in Figure $6 A$, the number of Fos-IR cells in the SCN of IGL-lesioned and sham-operated rats did not differ. Thus, under the lighting conditions of this experiment, the SCN of IGL-lesioned animals appeared to be as responsive to the light at dawn as that of the sham-operated animals.

To confirm that IGL lesions did not alter the response of the SCN to light during SPP housing, animals (from Experiment 2), 


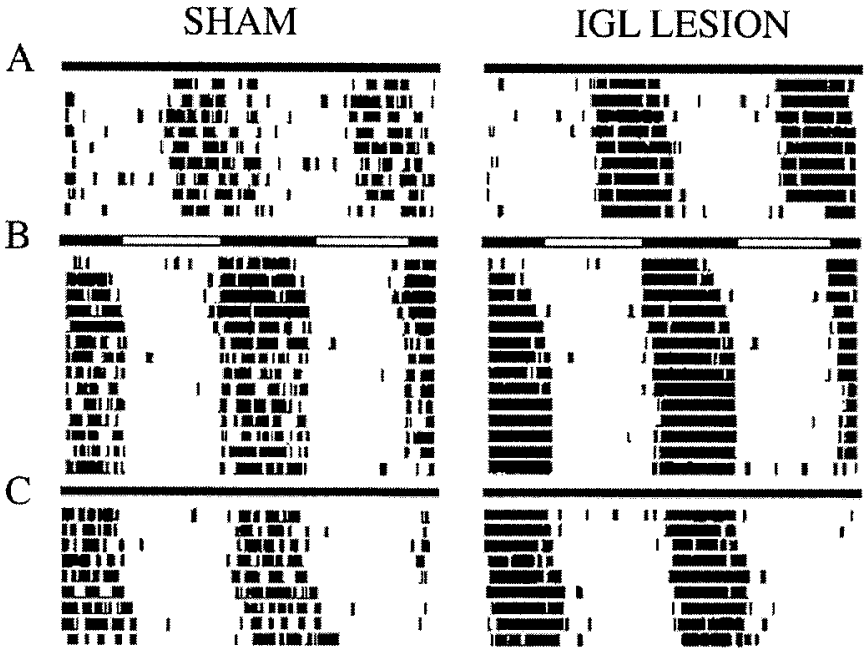

Figure 5. Double-plotted actograms of body temperature records of a sham-operated and an IGL-lesioned rat housed in DD $(A)$, LD12:12 $(B)$, and DD $(C)$. Light bars = lights on; dark bars = lights off.

initially entrained to LD12:12 and subsequently housed under an SPP for $4 \mathrm{~d}$, were killed on the fifth day after either the dawn or dusk light pulse. As shown in Figure $6 B$, the dawn pulse induced moderate Fos-IR in the SCN, whereas the dusk pulse induced very little Fos-IR, and no differences were noted between the groups. Thus, both the IGL-lesioned and the sham-operated animals in this study exhibited the pattern of Fos expression in the SCN previously seen in response to SPP light pulses in intact rats (Schwartz et al., 1994).

Finally, to explore the effect of IGL lesions on the phase response of the SCN to the light pulse used in these experiments, additional groups of IGL-lesioned $(n=8)$ and sham-operated $(n=4)$ rats were housed in DD for $24-36 \mathrm{hr}$ and then given the 1 hr light pulse between CT3 and CT5 or CT15 and CT17. No differences were observed between groups in mean number of SCN Fos-immunoreactive cells. All animals given the $1 \mathrm{hr}$ light pulse in the subjective night between CT15 and CT17 exhibited robust Fos expression in the SCN, whereas those given the light pulse between CT3 and CT5 in the subjective day showed few Fos-IR neurons (Fig. 6C). These data show that under the conditions of the present experiments the phase dependency of Fos expression in the SCN in response to the $1 \mathrm{hr}$ light pulse was not affected by IGL lesion. Inasmuch as the ability of light to induce Fos expression during the subjective night is related to its ability to induce phase shifts in rodents (Aronin et al., 1990; Kornhauser et al., 1990; Rusak et al., 1990; Wollnik et al., 1994), the present data suggest that the SCN cells of IGL-lesioned animals were capable of responding in a typical manner to a phase-shifting light stimulus. Examples of Fos expression observed in the SCN of IGL-lesioned and sham-operated animals killed after light exposure during SPP or DD housing are shown in Figure 7.

\section{DISCUSSION}

Circadian rhythms are synchronized to environmental LD cycles via transmission of photic information from the retina to the SCN (Card and Moore, 1991; Morin, 1994). The RHT is thought to be necessary for photic entrainment to occur; destruction of the RHT results in free-running rhythms despite the presence of photic time cues (Johnson et al., 1988). However, because at least some retinal ganglion cell axon collaterals that comprise the RHT send bif urcating axons that terminate in the IGL (Pickard, 1985), destruction of the RHT also damages light transmission to the IGL and hence transmission of photic information to the SCN along the GHT. It is therefore possible that both the RHT and IGL-GHT pathways are necessary for photic entrainment. Nevertheless, previous studies in IGL-lesioned hamsters have not provided support for a significant contribution of the GHT to photic entrainment (Pickard et al., 1987; Harrington and Rusak, 1988; Johnson et al., 1989; Moore and Card, 1994; Harrington, 1997). The results of the present experiments suggest that in rats the IGL-GHT plays a critical role in entrainment to an SPP, a lighting schedule like that experienced by nocturnal rodents in the natural environment (DeCoursey, 1986). Rats and other nocturnal animals entrain normally to this lighting schedule (Pittendrigh and Daan, 1976; Rosenwasser et al., 1983; Stephan, 1983; DeCoursey, 1986). Indeed, if housed in simulated den cages, animals emerge from their darkened dens near dawn and dusk, and rhythms are reset according to such light exposures (DeCoursey, 1986). In the present study, IGL-ablated rats exhibited freerunning rhythms for at least 3 weeks after release into SPP housing, despite showing normal entrainment to LD12:12. These animals exhibited normal profiles of Fos expression in the SCN in response to the entraining light pulse used in these experiments whether they were maintained under entrained or free-running conditions. These findings suggest that the effects of IGL lesions on entrainment to the SPP were not caused by a disruption in the transmission of the entraining light pulse to the SCN.

The failure to entrain to SPP housing after IGL lesion cannot be easily explained. One possibility is that IGL lesions alter the phase response curve (PRC) to light such that the daily phase shifts required to entrain to a $24 \mathrm{hr}$ SPP are unattainable. In hamsters, there is evidence that IGL lesions alter the response to phase-shifting light pulses, leading to smaller phase advances (Harrington and Rusak, 1986; Pickard et al., 1987) and larger phase delays (Pickard et al., 1987), although this was found only at circadian times when light pulses induce maximal advance and delay shifts. In normal intact rats, the phase delay portion of the PRC is larger than the phase advance portion (Summer and McCormack, 1982). If, in rats, IGL lesions were to shift the PRC in the same direction as in the hamster, then the $24 \mathrm{hr}$ SPP might be outside the limits of entrainment (Daan and Pittendrigh, 1976). Our finding, however, that IGL-lesioned rats show normal profiles of Fos expression in response to the entraining light pulse at different circadian phases, and that they maintain a normal circadian range of free-running periods, indicates that if IGL lesions have an effect on period or PRC, it is small.

One question that arises out of the present work is whether and how masking of overt rhythmicity normally contributes to stable entrainment of the clock to the $24 \mathrm{hr}$ day. An understanding of this might help explain why IGL-lesioned rats can entrain to a normal LD cycle. It has been suggested that the acute masking effect of light on rhythmic variables complements the regulation of those variables by the clock (Mrosovsky, 1994). If this were true, then attenuation of the masking effects of light would eliminate an important element of the entrainment mechanism. Although hamsters with IGL lesions are less active than intact animals (Janik and Mrosovsky, 1994; Wickland and Turek, 1994), no differences between intact and IGL-lesioned hamsters or rats have been observed with respect to light-induced suppression of activity and body temperature (rats: present study; hamsters: $\mathrm{U}$. Redlin, N. Vrang, and N. Mrosovsky, unpublished observations). These findings indicate that the masking effects of light persist 


\section{$\square$ SHAM $\square \quad$ LESION}
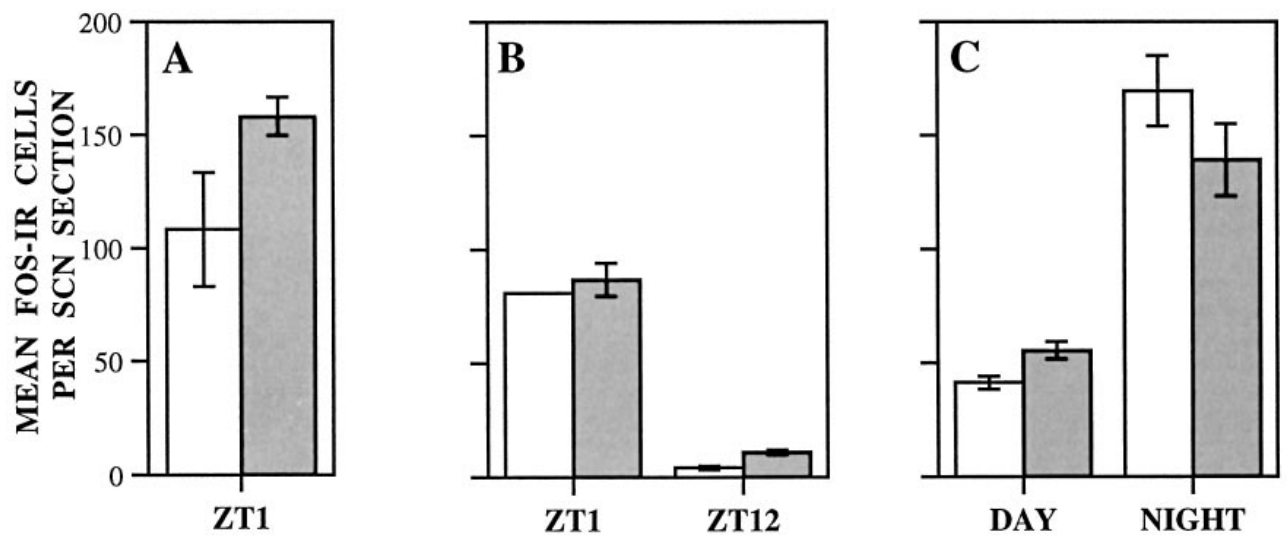

Figure 6. Mean number of Fos-immunoreactive cells per SCN section in IGL-lesioned and sham-operated rats. $A$, animals housed under LD12:12 and killed $1 \mathrm{hr}$ after light onset $(Z T 1)$. No difference between groups $\left(t_{(6)}=2.308, \mathrm{NS}\right)$. B, Animals housed under SPP and killed after the dawn $(Z T 1)$ or dusk (ZT12) light pulse. (ANOVA not performed because of small number of sham-operated animals.) $C$, Animals housed in DD and killed after a 1 hr light pulse during the subjective day $\left(\right.$ CT3-CT5) or subjective night $\left(\right.$ CT15-CT17). Significant effect for time of day $\left(F_{(1,8)}=64.664 ; p<0.001\right)$.

after lesion. Indeed, a recent study has shown that IGL-lesioned hamsters may be even more sensitive to the masking effects of light than intact animals (Redlin, Vrang, and Mrosovsky unpublished observations). It is possible, therefore, that in IGLlesioned animals feedback to the circadian clock, resulting from light-induced suppression of activity, facilitates phase shifts and results in better entrainment under full photoperiods than under the SPP.

A related issue is the contribution of nonphotic cues to stable entrainment. Several lines of research support a role for the IGL in nonphotic phase resetting of the circadian clock. In hamsters and mice, nonphotic stimuli induce phase shifts characterized by large phase advances during the subjective day; these phase shifts are prevented in IGL-lesioned animals (Biello et al., 1991; Meyer et al., 1993; Wickland and Turek, 1994; Janik and Mrosovsky, 1994; Marchant et al., 1997; Maywood et al., 1997) (for review, see Mrosovsky, 1995; Harrington, 1997). Furthermore, infusions of NPY into the SCN and electrical stimulation of the IGL both induce phase shifts in a pattern similar to those induced by nonphotic stimuli (cf. Mrosovsky, 1995) and different from those induced by light (Albers and Ferris, 1984; Rusak et al., 1989). The IGL has been proposed as the site of integration of photic and nonphotic stimuli that influence the circadian clock (Moore and Card, 1994; Mrosovsky, 1995, 1996; Harrington, 1997). That exposure to nonphotic stimuli can alter circadian responses to photic stimuli or LD cycles is known (Mrosovsky, 1996); the mechanisms underlying such interactions are not. A recent study using an SPP has demonstrated the importance of nonphotic cues in entrainment. Under SPP housing, hamsters exposed to a novel running wheel during the subjective day exhibited phase jumps in the entrained activity rhythm, which resulted in a switch in subjective day and night times (Sinclair and Mistlberger, 1997). Thus, if in rats the IGL mediates the transmission of nonphotic signals to the circadian clock, and if such signals enhance photic entrainment as they do in hamsters, then IGL-lesioned rats may lack the nonphotic input necessary to support stable entrainment under the conditions of an SPP.

The absence of NPY turnover in the SCN after complete, bilateral IGL ablation (Moore and Card, 1994; Harrington, 1997) likely contributes to the failure of IGL-lesioned animals to en- train to the SPP. Electrical stimulation of the IGL and NPY infusion into the SCN region have been shown to induce phase shifts in circadian activity rhythms with a PRC that resembles the phase-shifting effects of nonphotic stimuli (Albers and Ferris, 1984; Rusak et al., 1989). NPY can suppress excitatory neurotransmission in the SCN as well as induce phase-dependent phase shifts in SCN neuronal activity in vitro (Gribkoff et al., 1998). Although NPY-induced inhibition of excitatory neurotransmission is independent of circadian phase during the subjective day (Gribkoff et al., 1998), the fact that release of NPY in the SCN peaks during transition times, and that exogenous NPY can modulate both glutamate- and light-induced phase shifts, suggests that NPY may alter the responsiveness of SCN neurons to light as a function of circadian phase (Shinohara et al., 1993; van den Pol et al., 1996; Biello et al., 1997; Weber and Rea, 1997).

In the present experiments, lesions of the IGL were associated with considerable damage to several areas of the brain including parts of the hippocampus and LGN. One cannot exclude the possibility that such damage may have contributed to the inability of these animals to entrain to the SPP. The hippocampus has been implicated in the photoperiodic response of hamsters housed under short days; hippocampal damage reduces the extent of testicular regression during short photoperiod exposure (Smale and Morin, 1990). In addition, hippocampal damage impairs performance in light/dark discrimination tasks (for review, see Eichenbaum, 1996). Similarly, ventral LGN damage has been found to impair performance on brightness discrimination tasks (cf. Harrington, 1997). The dorsal LGN relays visual information to higher cortical regions and is involved in saccadic suppression, a mechanism underlying visual attention (Sherman and Koch, 1986; Burr et al., 1994). In addition, the LGN may also integrate photic information from both the circadian and visual systems; perhaps such input to the SCN is necessary for entrainment to discrete pulses of light. However, animals treated neonatally with monosodium glutamate, who sustain extensive neurotoxic damage to the visual system and the hippocampus (Kizer et al., 1978), exhibit stable entrainment to $24 \mathrm{hr}$ SPP lighting schedules (our unpublished observations).

Finally, IGL lesions result in destruction not only of the GHT but also the hypothalamogeniculate projection originating in the 


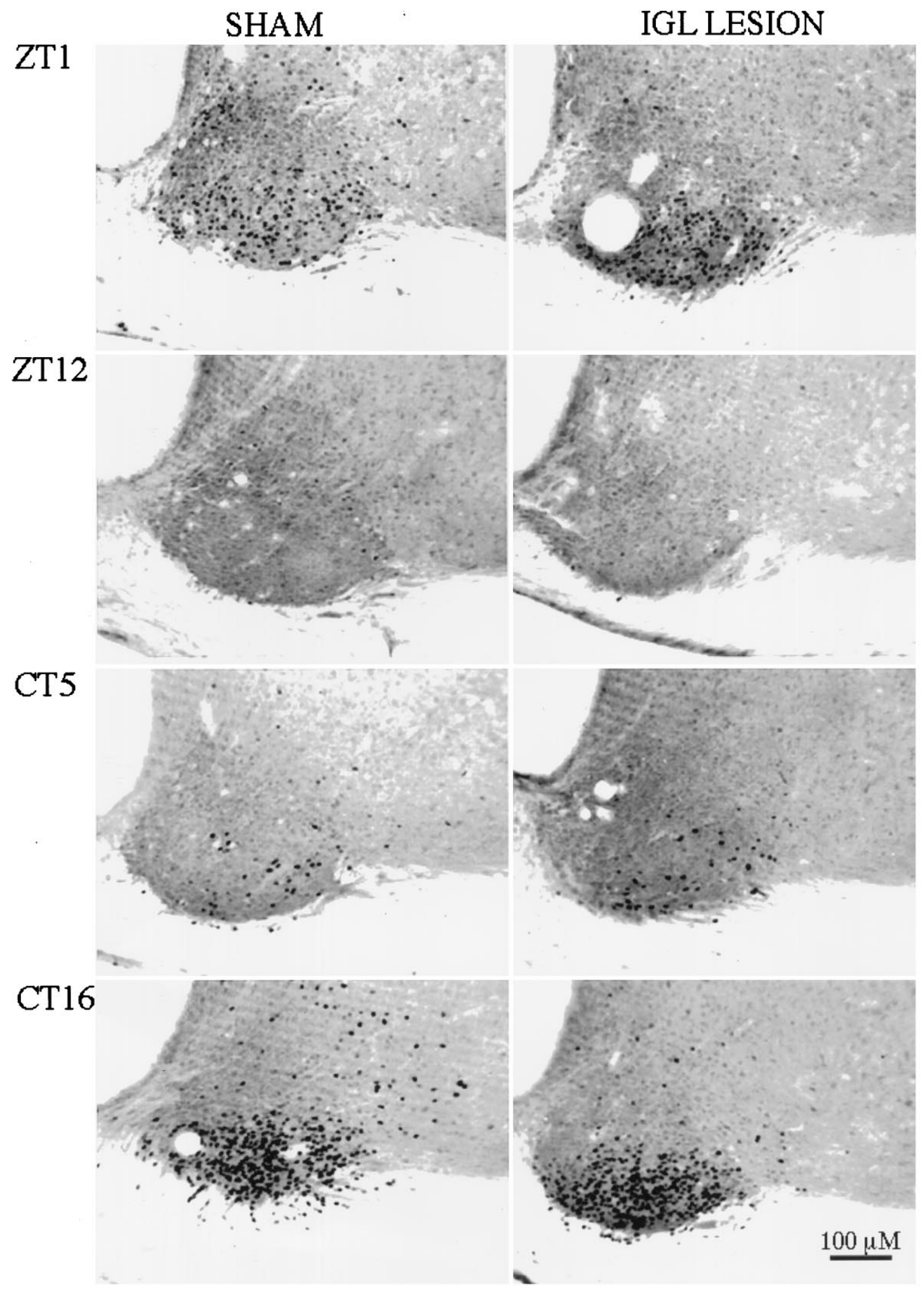

Figure 7. Fos immunoreactivity in the SCN of rats (left, SHAM; right, IGL-LESION) after light exposure at ZT0-1 or ZT11-12 of an SPP (top) or at CT4-CT5 or CT15-CT16 during DD (bottom). 
dorsomedial region of the SCN and terminating in the IGL (Watts et al., 1987). The function of this pathway in the circadian system is not known. One cannot exclude the possibility, therefore, that damage to dorsomedial SCN neurons whose fibers terminate in the IGL may have contributed to these findings.

In summary, the results of the present study show that the IGL-GHT is involved in photic entrainment of circadian temperature rhythms to a $24 \mathrm{hr}$ skeleton photoperiod in the rat. It is likely that NPY neurotransmission in the SCN contributes to the response of the circadian clock to light during twilight times, and that the absence of such input in the lesioned animal renders the circadian system incapable of entraining to discrete pulses of light. Whether masking enhances phase resetting of the circadian clock under full photoperiods, and the contribution of nonphotic cues to photic entrainment, are important questions for future research.

\section{REFERENCES}

Albers HE, Ferris CF (1984) Neuropeptide Y: role in light-dark entrainment of hamster circadian rhythms. Neurosci Lett 50:163-168.

Aronin N, Sagar SM, Sharp FR, Schwartz WJ (1990) Light regulates expression of a Fos-related protein in rat suprachiasmatic nuclei. Proc Natl Acad Sci USA 87:5959-5962.

Biello SM, Harrington ME, Mason R (1991) Geniculo-hypothalamic tract lesions block chlordiazepoxide-induced phase advances in Syrian hamsters. Brain Res 552:47-52.

Biello SM, Golombek DA, Harrington ME (1997) Neuropeptide Y and glutamate block each other's phase shifts in the suprachiasmatic nucleus in vitro. Neuroscience 77:1049-1057.

Burr DC, Morrone MC, Ross J (1994) Selective suppression of the magnocellular visual pathway during saccadic eye movements. Nature 371:511-513.

Card JP, Moore RY (1991) The organization of visual circuits influencing the circadian activity of the suprachiasmatic nucleus. In: Suprachiasmatic nucleus: the mind's clock (Klein DC, Moore RY, Reppert SM, eds), pp 51-76. New York: Oxford UP.

Daan S, Pittendrigh CS (1976) A functional analysis of circadian pacemakers in nocturnal rodents. II. The variability of phase response curves. J Comp Physiol 106:253-266.

Dark JG, Asdourian D (1975) Entrainment of the rat's activity rhythm by cyclic light following lateral geniculate nucleus lesions. Physiol Behav 15: 295-301.

de Togni P, Niman H, Raymond V, Sawchenko P, Verma IM (1988) Detection of fos protein during osteogenesis by monoclonal antibodies. Mol Cell Biol 8:2251-2256.

DeCoursey PJ (1986) Light-sampling behavior in photoentrainment of a rodent circadian rhythm. J Comp Physiol [A] 159:161-169.

Edelstein K, Amir S (1996) Constant light induces persistent Fos expression in rat intergeniculate leaflet. Brain Res 731:221-225.

Edelstein K, Amir S (1997) The intergeniculate leaflet is necessary for entrainment to a skeleton photoperiod. Soc Neurosci Abstr 23:791.

Edelstein K, Amir S (1999) The intergeniculate leaflet does not mediate the disruptive effects of constant light on circadian rhythms in the rat. Neuroscience, in press.

Eichenbaum H (1996) Is the rodent hippocampus just for "place"? Curr Opin Neurobiol 6:187-195.

Gribkoff VK, Pieschl RL, Wisialowski TA, van den Pol AN, Yocca FD (1998) Phase shifting of circadian rhythms and depression of neuronal activity in the rat suprachiasmatic nucleus by neuropeptide Y: mediation by different receptor subtypes. J Neurosci 18:3014-3022.

Harrington ME (1997) The ventral lateral geniculate nucleus and the intergeniculate leaflet: interrelated structures in the visual and circadian systems. Neurosci Biobehav Rev 21:705-727.

Harrington ME, Rusak B (1986) Lesions of the thalamic intergeniculate leaflet alter hamster circadian rhythms. J Biol Rhythms 1:309-325.

Harrington ME, Rusak B (1988) Ablation of the geniculo-hypothalamic tract alters circadian activity rhythms of hamsters housed under constant light. Physiol Behav 42:183-189.

Janik D, Mrosovsky N (1994) Intergeniculate leaflet lesions and behaviorally-induced shifts of circadian rhythms. Brain Res 651:174-182.
Johnson RF, Moore RY, Morin LP (1988) Loss of entrainment and anatomical plasticity after lesions of the hamster retinohypothalamic tract. Brain Res Bull 460:297-313.

Johnson RF, Moore RY, Morin LP (1989) Lateral geniculate lesions alter circadian activity rhythms in the hamster. Brain Res Bull 22:411-422.

Kizer JS, Nemeroff CB, Youngblood WW (1978) Neurotoxic amino acids and structurally related analogs. Pharmacol Rev 29:301-318.

Kornhauser JM, Nelson DE, Mayo KE, Takahashi JS (1990) Photic and circadian regulation of c-fos gene expression in the hamster suprachiasmatic nucleus. Neuron 5:127-134.

Marchant EG, Watson NV, Mistlberger RE (1997) Both neuropeptide Y and serotonin are necessary for entrainment of circadian rhythms in mice by daily treadmill running schedules. J Neurosci 17:7974-7987.

Maywood ES, Smith E, Hall SJ, Hastings MH (1997) A thalamic contribution to arousal-induced, non-photic entrainment of the circadian clock of the Syrian hamster. Eur J Neurosci 9:1739-1747.

Meyer EL, Harrington ME, Rahmani T (1993) A phase-response curve to the benzodiazepine chlordiazepoxide and the effect of geniculohypothalamic tract ablation. Physiol Behav 53:237-243.

Moore RY (1983) Organization and function of a central nervous system circadian oscillator: the suprachiasmatic hypothalamic nucleus. Fed Proc 42:2783-2789.

Moore RY, Card JP (1994) Intergeniculate leaflet: an anatomically and functionally distinct subdivision of the lateral geniculate complex. J Comp Neurol 344:403-430.

Morin LP (1994) The circadian visual system. Brain Res Rev $67: 102-127$.

Mrosovsky N (1994) In praise of masking: behavioral responses of retinally degenerate mice to dim light. Chronobiol Int 11:343-348.

Mrosovsky N (1995) A non-photic gateway to the circadian clock of hamsters. Circadian clocks and their adjustment. CIBA Found Symp 183:154-174.

Mrosovsky N (1996) Locomotor activity and non-photic influences on circadian clocks. Biol Rev 71:343-372.

Paxinos G, Watson C (1998) The rat brain in stereotaxic coordinates, Ed 4. San Diego: Academic.

Pickard GE (1985) Bifurcating axons of retinal ganglion cells terminate in the hypothalamic suprachiasmatic nucleus and the intergeniculate leaflet of the thalamus. Neurosci Lett 55:211-217.

Pickard GE (1989) Entrainment of the circadian rhythm of wheelrunning activity is phase shifted by ablation of the intergeniculate leaflet. Brain Res 494:151-154.

Pickard GE (1994) Intergeniculate leaflet ablation alters circadian rhythms in the mouse. NeuroReport 5:2186-2188.

Pickard GE, Ralph MR, Menaker M (1987) The intergeniculate leaflet partially mediates effects of light on circadian rhythms. J Biol Rhythms 2:35-56.

Pittendrigh CS, Daan S (1976) A functional analysis of circadian pacemakers in nocturnal rodents. IV. Entrainment: pacemaker as clock. J Comp Physiol 106:291-331.

Rea MA (1989) Light increases Fos-related protein immunoreactivity in the rat suprachiasmatic nuclei. Brain Res Bull 23:577-581.

Roenneberg T, Foster RG (1997) Twilight times: light and the circadian system. Photochem Photobiol 66:549-562.

Rosenwasser AM, Boulos Z, Terman M (1983) Circadian feeding and drinking rhythms in the rat under complete and skeleton photoperiods. Physiol Behav 30:353-359.

Rusak B, Meijer JH, Harrington ME (1989) Hamster circadian rhythms are phase-shifted by electrical stimulation of the geniculo-hypothalamic tract. Brain Res 493:283-291.

Rusak B, Robertson HA, Wisden W, Hunt SP (1990) Light pulses that shift rhythms induce gene expression in the suprachiasmatic nucleus. Science 248:1237-1240.

Schwartz WJ, Takeuchi J, Shannon W, Davis EM, Aronin N (1994) Temporal regulation of light-induced Fos and Fos-like protein expression in the ventrolateral subdivision of the rat suprachiasmatic nucleus. Neuroscience 58:573-583.

Sherman SM, Koch C (1986) The control of retinogeniculate transmission in the mammalian lateral geniculate nucleus. Exp Brain Res 63:1-20.

Shinohara K, Tominaga K, Fukuhara C, Otori Y, Inouye S-IT (1993) Processing of photic information within the intergeniculate leaflet of the lateral geniculate body: assessed by neuropeptide Y immunoreactivity in the suprachiasmatic nucleus of rats. Neuroscience 56:813-822. Sinclair SV, Mistlberger RE (1997) Scheduled activity reorganizes cir- 
cadian phase of Syrian hamsters under full and skeleton photoperiods. Behav Brain Res 87:127-137.

Smale L, Morin LP (1990) Photoperiodic responsiveness of hamsters with lesions of the lateral geniculate nucleus is related to hippocampal damage. Brain Res Bull 24:185-190.

Stephan FK (1983) Circadian rhythms in the rat: constant darkness, entrainment to $\mathrm{T}$ cycles and to skeleton photoperiods. Physiol Behav 30:451-462.

Summer TL, McCormack CE (1982) Phase-response-curve (PRC) of circadian locomotor activity of the rat. Fed Proc 41:1698.

van den Pol AN, Obrietan K, Chen G, Belousov AB (1996) Neuropeptide Y-mediated long-term depression of excitatory activity in suprachiasmatic nucleus neurons. J Neurosci 16:5883-5895.
Watts AG, Swanson LW, Sanchez-Watts G (1987) Efferent projections of the suprachiasmatic nucleus: I. Studies using anterograde transport of phaseolus vulgaris leucoagglutinin in the rat. J Comp Neurol 258:204-229.

Weber ET, Rea MA (1997) Neuropeptide Y blocks light-induced phase advances but not delays of the circadian activity rhythm in hamsters. Neurosci Lett 231:159-162.

Wickland C, Turek FW (1994) Lesions of the thalamic intergeniculate leaflet block activity-induced phase shifts in the circadian activity rhythm of the golden hamster. Brain Res 660:293-300.

Wollnik F, Brysch W, Uhlmann E, Gillardon F, Bravo R, Zimmerman M, Schlingensiepen KH, Herdegen T (1995) Block of c-Fos and JunB expression by antisense oligonucleotides inhibits light-induced phase shifts of the mammalian circadian clock. Eur J Neurosci 7:388-393. 